\title{
Number and Nature of Incidental Findings in Cone-beam Computed Tomography Made for Oral Surgery Purposes
}

\author{
Fatima M Jadu
}

\begin{abstract}
Aim: Oral and maxillofacial surgeons (OMFSs) request cone-beam computed tomography (CBCT) examinations more than any other dental specialty. The aim of the current study was to determine the frequency and type of incidental findings (IFs) from CBCT examinations specifically requested by OMFSs.

Materials and methods: The database of a university-based CBCT service was reviewed for examinations requested by an OMFS over a 1-year period. The indications for the CBCT request ranged from implant and impaction to trauma-related causes. A board-certified oral and maxillofacial radiologist (OMFR) reviewed the CBCT images and the collected data were analyzed for descriptive results.

Results: The frequency of IFs was $78 \%$. The greatest number of IFs was found in the paranasal region followed by the cervical vertebrae and the nasopharyngeal area. Most IFs were insignificant but nearly half the cases (48\%) required a follow-up or referral appointment.

Conclusion: IFs from CBCT examinations requested by OMFSs are common and a substantial number required follow-up or referral.

Clinical significance: The results of the current study highlight the importance of examining the entire volume of CBCT examinations and reporting any and all IFs. This, in turn, requires adequate knowledge and training in CBCT image interpretation or, alternatively, effective collaboration with oral and maxillofacial radiologists.

Keywords: Cone-beam computed tomography, Incidental findings, OMFR, Oral and maxillofacial surgeon.

World Journal of Dentistry (2019): 10.5005/jp-journals-10015-1659
\end{abstract}

\section{INTRODUCTION}

In dentistry, unlike medicine, dentists are trained to acquire and interpret their own diagnostic images. In the past, these diagnostic images consisted largely of two-dimensional (2D) conventional radiographs, most of which were intraoral (e.g., periapical and bitewing radiographs). Others were extraoral images including, but not limited to, panoramic and cephalometric radiographs. Dentists and dental specialists continue to rely on these diagnostic images. However, the past 10 years have witnessed a paradigm shift toward the utilization of cone-beam computed tomography (CBCT). The widespread availability of this 3D diagnostic imaging system is due to the many advantages that it offers and the various indications it has fulfilled in the maxillofacial area.

With the prevalent use of $C B C T$, two concerns have surfaced. The first is the inappropriate acquisition of CBCT images and this is due to the lack of customized exposure parameters. The second is the incomplete analysis of CBCT images resulting in missed incidental findings (IFs). IFs are defined as "imaging findings that are not related to the clinical indication for which the imaging was performed". ${ }^{1}$ The problem of missed IFs is further compounded by distraction of the clinicians by points of interest in a diagnostic image. $^{2}$

Many studies have been published in the last few years to address concerns of missed IFs in CBCT examinations. Some authors have tailored their analysis to $C B C T$ examinations of various field-ofview (FOV) settings; others analyzed CBCT examinations according to their indications. ${ }^{1,3,4}$ Yet, no data are available regarding CBCT scans made for oral and maxillofacial surgery (OMFS) purposes despite the fact that OMFS has been consistently shown to request CBCT scans the most. ${ }^{5}$ The three most common indications for which OMFS request $C B C T$ scans are the following: pre-dental implant planning, impaction assessment, and trauma evaluation. ${ }^{5}$
Department of Oral Diagnostic Sciences, Faculty of Dentistry, King Abdulaziz University, Jeddah, Kingdom of Saudi Arabia

Corresponding Author: Fatima M Jadu, Department of Oral Diagnostic Sciences, Faculty of Dentistry, King Abdulaziz University, Jeddah, Kingdom of Saudi Arabia, Phone: +966 254 00000, e-mail: fjadu@kau. edu.sa

How to cite this article: Jadu FM. Number and Nature of Incidental Findings in Cone-beam Computed Tomography Made for Oral Surgery Purposes. World J Dent 2019;10(5):390-392.

Source of support: Nil

Conflict of interest: None

Therefore, the aim of the current study was to determine the frequency and type of IFs in CBCT examinations specifically requested by OMFS.

\section{Materials and Methods}

Ethical approval was obtained from the academic institution where the oral surgery and imaging services are based. Then the database of the CBCT imaging service was reviewed. All CBCT scans acquired during a 1-year time period and requested by an OMFS were reviewed. The same academic institution at which the imaging service was based employed all of the referring surgeons. Inclusion criteria were cases made for pre-dental implant planning, impaction assessment, and trauma evaluation. Cases referred for other reasons, such as pathology investigation, were excluded. Additionally, caries, periodontal disease, and missing third molars were not considered to be IFs.

Fortunately, all patients were scanned in the same iCAT Classic Scanner (Imaging Sciences International, Hatfield, Pennsylvania, USA).

() The Author(s). 2019 Open Access This article is distributed under the terms of the Creative Commons Attribution 4.0 International License (https://creativecommons. org/licenses/by-nc/4.0/), which permits unrestricted use, distribution, and non-commercial reproduction in any medium, provided you give appropriate credit to the original author(s) and the source, provide a link to the Creative Commons license, and indicate if changes were made. The Creative Commons Public Domain Dedication waiver (http://creativecommons.org/publicdomain/zero/1.0/) applies to the data made available in this article, unless otherwise stated. 
Exposure parameters were set according to the manufacturer's recommendations but the FOV varied and three settings were used $(16 \times 13 \mathrm{~cm}, 16 \times 11 \mathrm{~cm}$, or $16 \times 10 \mathrm{~cm})$. The resolution of the images also varied according to the voxel (VOX) size setting, which was either $0.4 \mathrm{~mm}$ or $0.3 \mathrm{~mm}$.

A single board-certified radiologist reviewed the CBCT images using the Vision Software (Imaging Sciences International, Hatfield, Pennsylvania, USA). No more than 10 cases were reviewed per day to avoid eye fatigue. The first 10 cases were evaluated again after 1 week to assess intraexaminer reliability. In addition to patients' demographic data, the number, location, and nature of the IFs were recorded.

Statistical analysis was performed using SPSS (SPSS Inc, Chicago, Illinois, USA) version 23. Simple descriptive statistics were calculated using counts and percentages. Intraexaminer reliability was assessed using kappa statistics.

\section{Results}

The number of CBCT scans included in this study was 512 . The patients for whom the scans were made ranged in age between 14 years and 89 years with a mean age of 34.7 years. Females constituted $57 \%$ of cases and males constituted $43 \%$.

The total number of IFs was 838 and these were identified in 399 cases. That translates to a frequency of $78 \%$ or approximately $1.5 \mathrm{IFs}$ per scan. The intraobserver kappa score was 1.0, which is considered indicative of perfect agreement.

The IFs were categorized into one of six groups based on anatomical region as per the methods developed by Edwards et al. ${ }^{1}$ These results are presented in Table 1. The total number of cases does not amount to a perfect $100 \%$ because some cases had more than one IF. The same IFs were also categorized, more practically, into three groups according to level of importance as described by Barghan et al. ${ }^{3}$ The three levels of importance were the following: IF requiring referral, IF requiring follow-up, and insignificant IF (Table 2). Cases were categorized according to the IF with the highest level of importance.

\section{Discussion}

The frequency of IFs in the current study was $78 \%$, which lies in the middle of the range of frequencies reported by other published

Table 1: Frequency of CBCT IFs categorized by anatomic location

\begin{tabular}{ll}
\hline Anatomic region & Frequency of IFs ( $n=838)(\%)$ \\
\hline Dentoalveolar & $210(25)$ \\
Paranasal sinuses & $243(29)$ \\
Nasopharyngeal airway & $100(12)$ \\
Surrounding hard/soft tissues & $134(16)$ \\
Temporomandibular joints & $74(9)$ \\
Cervical vertebrae & $184(22)$ \\
\hline
\end{tabular}

$\mathrm{CBCT}$, cone-beam computed tomography; IFs, incidental findings

Table 2: Frequency of CBCT IFs categorized by importance

\begin{tabular}{ll}
\hline Importance level of IFs & Frequency $(n=399)(100 \%)$ \\
\hline Referral & $64(16.0)$ \\
Follow-up & $128(32.0)$ \\
Insignificant & $207(51.8)$ \\
\hline
\end{tabular}

IFs, incidental findings studies. ${ }^{3,6-8}$ Reported frequencies range from as low as $24.5 \%$ Cha et al. to as high as $99 \%$ Theodoridis et al. ${ }^{8,9}$ This great variation in reported frequencies is best explained by Edwards et al. as being due to variations in patient samples, radiologist reporting style, and/or the definition of an IF.

The majority of IFs were insignificant. However, a substantial number required follow-up or referral, which is consistent with Bargh et al. ${ }^{3}$ Contrary to the current study, the number of cases that required referral was greater in Bargh et al.'s study (31\% compared to $16 \%$ in the current study). ${ }^{3}$ Cases that required referral included base of skull lesions, jaw lesions, and cases with suspected airway compromise. Follow-up was recommended for cases such as severe mucositis in the paranasal sinuses, adenoid hyperplasia, and degenerative changes of the temporomandibular joints (TMJs) and cervical vertebrae after clinical correlation. Regardless of the action required, this result highlights the importance of recognizing and reporting IFs, which are often the first sign of a condition and may significantly affect patient care.

The most common dentoalveolar IF was dense bone island (DBI) ( $57 \%$ of 210 IF). This was closely following by apical inflammatory conditions (34\% of 210 IF). This result was consistent with the findings of Drage et al. ${ }^{10}$ The third most common finding in the dentoalveolar area was periapical cemento-osseous dysplasia (15\% of 210 IF). These results are different from Edwards et al., where hypodontia was the most common IF in the dentoalveolar area followed by impactions and then DBI. ${ }^{1}$ We believe this to be a reflection of the patient population studied which was younger in Edwards et al. ${ }^{1}$ Barghan et al. studies a group of patients similar in age to the current study but did not comment on IFs in the dentoalveolar area. $^{3}$

The greatest number of IFs was found in the paranasal sinus area, especially the maxillary sinuses ( $92 \%$ of IF in the paranasal sinuses). This is not surprising considering the high occurrence of conditions such as mucositis and retention pseudocysts, both of which are reported frequently in the maxillary sinuses in all forms of imaging. ${ }^{11,12}$ These results are in general agreement with the results of others such as Edwards et al.' Categorizing the paranasal, IFs was challenging because conditions like these require corroboration with clinical signs and symptoms. This emphasizes the importance of active collaboration and efficient communication between the various dental disciplines in order to provide the best patient care.

Dystrophic calcifications of the tonsils were the most common IF in the nasopharyngeal airway region (44\% of 100 IFs). This result is unique to the current study and warrants further investigation. The second most common finding was adenoid hyperplasia (30\% of 100 IF). This is in agreement with Barghan et al. ${ }^{3}$ They found narrowing of the pharyngeal airway to be the most common IF followed by adenoid hyperplasia. ${ }^{3}$ In contrast, most other studies found adenoid hyperplasia to be the most common IF in the nasopharyngeal area. ${ }^{1,9,13}$ Other IFs in the nasopharyngeal area included tonsillar hypertrophy, nasal polyps, nasal septum deviation, and rhinolith.

Soft tissue calcifications constituted the bulk of the IFs in the surrounding soft tissue ( $72 \%$ of $134 \mathrm{IFs}$ ). Calcifications such as sialoliths, lymph node calcifications, and vascular calcifications were noted. The frequency of these findings varies greatly among other publications. ${ }^{1,7,13}$ More important is the significance of these calcifications, which is still largely controversial. We believe it to be imperative to include these findings in imaging reports until a robust consensus is reached regarding their importance. Another noteworthy finding was elongation of the styloid process 
and/or calcification of the stylohyoid ligament (33\% of 134 IFs). This may seem like an indolent IF when, in fact, it can be the cause of symptoms in patients and, therefore, should be taken seriously and correlated with clinical findings. ${ }^{14}$

IFs related to the TMJs and the cervical vertebrae were surprisingly common constituting nearly one-third of the IFs (31\% of all IF). ${ }^{1}$ Sclerosis (54\%) and flattening (44\%) were the two most common findings followed by osteophyte formation (34\%) and surface erosion (29\%). The former two IF were considered changes consistent with remodeling while the latter two were considered degenerative changes. The frequency of IF in percentage was calculated based on a total of $258 \mathrm{IFs}$ in the TMJs and cervical vertebrae areas combined. These results are in agreement with some publications including Allareddy et al. and Barghan et al. ${ }^{3,13}$ Other studies reported a much lower frequency of degenerative changes. ${ }^{1,9}$ The variation among published studies is once again most likely a reflection of the population examined; specifically, the age factor because degenerative changes are more common in older patient populations. ${ }^{15}$

One observer reviewed all of the CBCT scans and this is a limitation of the current study. Future studies should collect data points from at least two observers. Also, no clinical correlation or follow-up was attempted because these were beyond the scope of this paper. The effect of image resolution on the detection of IFs is an interesting future direction, as is assessing the ability of dentists and dental specialists to identify IFs. Most importantly, future research should examine the impact of these IFs on patient management and treatment outcomes.

\section{Conclusion}

IFs from CBCT examinations requested by OMFSs are common and a substantial number required follow-up or referral.

\section{Clinical Significance}

The results of the current study highlight the importance of examining the entire volume of CBCT images and reporting any and all IFs. This, in turn, requires adequate knowledge and training in CBCT image interpretation or, alternatively, effective collaboration with oral and maxillofacial radiologists.

\section{References}

1. Edwards R, Altalibi M, Flores-Mir C. The frequency and nature of incidental findings in cone-beam computed tomographic scans of the head and neck region: a systematic review. J Am Dent Assoc 2013;144(2):161-170. DOI: 10.14219/jada.archive.2013.0095.

2. Kuhlberg AJ, Norton LA. Pathologic findings in orthodontic radiographic images. Am J Orthod Dentofacial Orthop 2003; 123(2):182-184. DOI: 10.1067/mod.2003.4.

3. Barghan S, Tahmasbi Arashlow M, Nair MK. Incidental findings on cone beam computed tomography studies outside of the Maxillofacial Skeleton. Int J Dent 2016;2016:9196503.

4. Oser DG, Henson BR, Shiang EY, et al. Incidental findings in small field of view cone-beam computed tomography scans. J Endod 2017;43(6):901-904. DOI: 10.1016/j.joen.2017.01.033.

5. Warhekar S, Nagarajappa S, Dasar PL. Incidental findings on cone beam computed tomography and reasons for referral by dental practitioners in indore city (m.p). J Clin Diagn Res 2015;9(2): ZC21-ZC24.

6. Edwards R, Alsufyani N, Heo G, et al. The frequency and nature of incidental findings in large-field cone beam computed tomography scans of an orthodontic sample. Prog Orthod 2014;15:37. DOI: 10.1186/ s40510-014-0037-x.

7. Price JB, Thaw KL, Tyndall DA, et al. Incidental findings from cone beam computed tomography of the maxillofacial region: a descriptive retrospective study. Clin Oral Implants Res 2012;23(11):1261-1268. DOI: 10.1111/j.1600-0501.2011.02299.x.

8. Theodoridis CVZ, Angelopoulos C. Incidental findings on CBCT and classification according to their significance. Oral Surg Oral Med Oral Pathol Oral Radiol 2019;127:47. DOI: 10.1016/j.00oo.2018.07.046.

9. Cha JY, Mah J, Sinclair P. Incidental findings in the maxillofacial area with 3-dimensional cone-beam imaging. Am J Orthod Dentofacial Orthop 2007;132(1):7-14. DOI: 10.1016/j.ajodo.2005.08.041.

10. Drage N, Rogers S, Greenall C, et al. Incidental findings on cone beam computed tomography in orthodontic patients. J Orthod 2013; 40(1):29-37. DOI: 10.1179/1465313312Y.0000000027.

11. Drumond JP, Allegro BB, Novo NF, et al. Evaluation of the prevalence of maxillary sinuses abnormalities through spiral computed tomography (CT). Int Arch Otorhinolaryngol 2017;21(2):126-133. DOI: 10.1055/ s-0036-1593834.

12. Vallo J, Suominen-Taipale L, Huumonen S, et al. Prevalence of mucosal abnormalities of the maxillary sinus and their relationship to dental disease in panoramic radiography: results from the health 2000 health examination survey. Oral Surg Oral Med Oral Pathol Oral Radiol Endod 2010;109:e80-e87. DOI: 10.1016/j.tripleo.2009.10.031.

13. Allareddy V, Vincent SD, Hellstein JW, et al. Incidental findings on cone beam computed tomography images. Int J Dent 2012;2012:871532. DOI: 10.1155/2012/871532.

14. Saccomanno S, Greco F, de Corso E, et al. Eagle's Syndrome, from clinical presentation to diagnosis and surgical treatment: a case report. Acta Otorhinolaryngol Ital 2018;38:166-169.

15. Alexiou K, Stamatakis H, Tsiklakis K. Evaluation of the severity of temporomandibular joint osteoarthritic changes related to age using cone beam computed tomography. Dentomaxillofac Radiol 2009; 38:141-147. DOI: 10.1259/dmfr/59263880. 